\title{
Pratiques transplateformes et convergence dans les usages des médias sociaux
}

\section{Mélanie Millette}

\section{(2) OpenEdition \\ 12 Journals}

Édition électronique

URL : http://journals.openedition.org/communicationorganisation/4116

DOI : 10.4000/communicationorganisation.4116

ISSN : $1775-3546$

Éditeur

Presses universitaires de Bordeaux

\section{Édition imprimée}

Date de publication : 1 juin 2013

Pagination : 47-58

ISBN : 978-2-86781-883-7

ISSN : $1168-5549$

\section{Référence électronique}

Mélanie Millette, "Pratiques transplateformes et convergence dans les usages des médias sociaux », Communication et organisation [En ligne], 43 | 2013, mis en ligne le 01 juin 2015, consulté le 01 mai 2019. URL : http://journals.openedition.org/communicationorganisation/4116; DOI : 10.4000/ communicationorganisation.4116 


\title{
Pratiques transplateformes et convergence dans les usages des médias sociaux
}

\author{
Mélanie Millette'
}

La réflexion que nous présentons ici a émergé au cours d'une recherche précédente sur les usages du podcasting indépendant (Millette), pratique qui consiste en la création de contenus sonores au formats variés (chronique, critique, sketch, playlist commentée, etc.), déposés sur Internet et rendus disponibles pour téléchargement ou écoute en ligne. À l'époque de cette recherche (2006 à 2009), les podcasters interrogés nous avaient confié observer un déplacement de la conversation avec leurs auditeurs : les commentaires, auparavant laissés directement sur le blog du podcaster, s'égrenaient de plus en plus dans diverses plateformes du Web participatif, notamment sur Facebook. De fait, pour mieux cerner le podcasting, il avait fallu considérer ce que nous nommons aujourd'hui les « pratiques transplateformes » des usagers et étendre le terrain à Twitter, Facebook, Last. fm et Blip. fm².

Dans nos recherches actuelles, soit pour notre projet de thèse et pour une étude de cas sur le podcasting ${ }^{3}$, nous observons que ces pratiques transplateformes continuent de gagner en complexité sur Internet. En parallèle, nous remarquons que cette propension à " partager » sur plusieurs plateformes en même temps constitue un point aveugle dans les travaux sur Internet et en sociologie des médias.

\footnotetext{
1 Mélanie Millette est doctorante en communication à l'Université du Québec à Montréal, coordonnatrice du Laboratoire de communication médiatisée par ordinateur (LabCMO) et membre du Groupe de recherche et d'observation sur les usages et cultures médiatiques (GRM). Boursière du Conseil de recherche en sciences humaines du Canada (CRSH) et de la Fondation Trudeau ; ses travaux de recherche portent sur la visibilité et la reconnaissance dans les médias sociaux, de même que sur les usages d'Internet dans les contextes jeunesse, sous-culturels et minoritaires ; millette.melanie@courrier.uqam.ca.

2 Réseau socionumérique strictement axé sur le partage de pièces musicales. Les usagers y sont identifiés comme des « DJs ». La plateforme Last.fm, qui vise aussi le partage musical, est également investie par certains podcasters.

3 Cette recherche du Laboratoire de communication médiatisée par ordinateur est soutenue par le Conseil de recherches en sciences humaines du Canada (CRSH), 2009-2012 et porte sur les formes sociales et la puissance d'agir des contributeurs dans les plateformes du Web social. L'auteure tient d'ailleurs à remercier Serge Proulx, Lorna Heaton, de même que les collègues ayant participé à cette recherche, pour leurs commentaires pertinents.
} 
Nous proposons ici de théoriser le concept de «pratique transplateforme ». Pourcefaire nous procéderons en trois temps. Nous définirons conceptuellement les pratiques transplateformes en mobilisant des travaux en Fans Studies, doublée d'une approche critique des industries industries culturelles. Puis, nous examinerons certains aspects de ces pratiques transplateformes et leurs implications dans les plateformes du Web social, notamment par rapport aux traces et au déplacement des communications. Nous situerons ces pratiques participatives dans les usages du Web social et nous illustrerons nos propos par des données tirées d'une étude de cas sur le podcasting. Finalement, nous mobiliserons le concept de trace pour analyser plus en profondeur ces pratiques. Nous conclurons en montrant que les contenus issus des pratiques transplateformes observées tendent à faire converger ces contenus vers le site de réseautage Facebook.

\section{Définir les pratiques transplateformes : tension entre les stratégies des industries culturelles et les usages des fans}

Le terme «transmédia ", de l'anglais transmedia, est abondamment utilisé dans les travaux sur les industries culturelles depuis le début des années 2000 pour qualifier le déploiement d'un récit de fiction sur divers supports médiatiques ${ }^{5}$. Il s'agit de diffuser des parcelles originales d'un scénario global sous diverses formes, par exemple de créer des webisodes et des podcasts qui viennent compléter le récit développé dans une série télévisée ou un film (Jenkins 2006a). Idéalement, les récits se complètent entre eux, sans redite, et chaque contenu diffusé tient compte des spécificités d'usage et des contingences du support médiatique choisi (par exemple privilégier les gros plans pour les webisodes qui seront vus sur appareils mobiles). Du point de vue de l'expérience de l'usager, ce type de narration transmédiatique installe une intertexualité par le biais d'un réseau de récits satellites arrimé au récit mère (Perryman2008).

L'approche transmédia est à distinguer de celle dite « cross-média », où un seul récit est simplement repris tel quel sur différents supports, comme c'est le cas avec des émissions de télévision rediffusées sur Internet. Au Québec, cette pratique est souvent appelée " multiplateforme ", notamment dans les stratégies médiatiques de la station d'État Radio-Canada.

4 Cette étude de cas est en cours : des entretiens exploratoires ont eu lieu, de même que l'analyse préliminaire de l'usage des médias sociaux et de la présence en ligne de trois podcasters.

5 L'un des premiers cas est la trilogie filmique The Matrix des frères Wachowski. Le premier film a pris l'affiche en 1999, le dernier en 2003 et l'ensemble de la stratégie transmédia entourant le lancement des films est devenue un exemple phare, incluant des jeux, des épisodes exclusifs à Internet, des sites dédiés, etc. Le cas de The Matrix est entre autres mentionné dans Perryman (2008), Evans (2008) et analysé en profondeur par Jenkins (2006a, chapitre 3).

6 À ne pas confondre avec « multimédia " qui qualifie l'intégration de plusieurs formes médiatiques (textes, sons, images). 
Les stratégies de diffusion transmédias dans les industries culturelles vont de pair avec le phénomène de la concentration des médias et la propension à tirer profit de l'engagement des fans envers une œuvre (Brooker 2001). Pour brosser un portrait rapide, et forcément partiel, on peut classer les approches de ces concepts en deux tendances, plus ou moins polarisées dans les travaux en sciences sociales. Se trouveraient d'un côté les tenants d'une approche critique des industries culturelles, et de l'autre les chercheurs en Fans Studies. Du point de vue critique, on souligne à juste titre que les pratiques transmédiatiques et cross-médiatiques vont de pair avec les stratégies de synergie des grands conglomérats et visent à tirer profit de l'engagement du public en exploitant leur attention à l'endroit d'un récit médiatique (Bouquillion et Matthews 2010). À l'opposé, les Fans Studies voient dans ces pratiques de l'industrie des opportunités pour les fans d'accroître leur influence ou, minimalement, de bénéficier de textes médiatiques plus variés et de plus nombreuses tribunes pour s'exprimer (Jenkins 2006a, 2006b ; Perryman 2008). Il n'est pas possible de témoigner ici des nuances présentes dans ces travaux, mais il se dégage néanmoins une tension entre entre les stratégies de l'industrie, d'une part, et les pratiques expressives des usagers, d'autre part. Cette tension est partie prenante de notre compréhension des pratiques transplateformes, mais avant de proposer une définition, il convient de resituer ces usages dans le contexte du Web social.

\section{Le contexte des médias sociaux}

Comme nous l'avons décrit ailleurs, les médias sociaux sont des supports médiatiques logiciels permettant aux usagers de maintenir une présence, de communiquer et d'interagir en ligne. [... Ces] dispositifs appuient et suscitent les échanges interactifs, de même que la communication interpersonnelle et de groupe. Ce faisant, ces dispositifs affectent la nature même des échanges. (Proulx, Millette et Heaton 2012 : 4). Les médias sociaux s'insèrent d'ailleurs dans le schéma capitaliste des entreprises qui tirent profit des avancées techniques et créatives pour monétiser les contributions (souvent gratuites) des internautes.

Les communications dans de tels dispositifs ont une portée exponentielle, qui laissent des traces parfaitement reproductibles. Dans les médias sociaux, la communication est cependant contrainte par les possibilités d'interactivité permises par les plateformes. Par exemple, Facebook a des options d'interactivité très fines, allant de la possibilité de signaler que l'on aime quelque chose à celle de laisser un commentaire, de publier un lien et de rediffuser une information affichée dans un statut d'usager. Plusieurs blogs personnels intègrent maintenant de telles options, favorisant la rediffusion et la rétroaction des commentateurs. De telles possibilités de rétroaction, qui s'incarnent sous la forme de divers textes médiatiques, donnent à l'observateur l'impression d'avoir sous les yeux un portrait co-construit lorsqu'il observe 
le profil d'une personne, un portrait où les commentaires des autres usagers viennent authentifier (ou contredire !) la présentation de soi proposée initialement. Comme nous le verrons plus loin dans l'étude de cas des podcasters Lada et George, l'identité numérique telle que déployée sur des plateformes de réseaux sociaux est une « coproduction où se rencontrent les stratégies des plateformes et les tactiques des utilisateurs » (Cardon2008: 98).

Les usages des médias sociaux se déploient donc dans un contexte particulièrement foisonnant. Les pratiques transplateformes viennent ajouter à la complexité de cette situation. Car lorsque l'on diffuse un élément sur une plateforme, il est désormais monnaie courante que des modalités de rediffusion vers d'autres sites soient arrimées à même l'interface, permettant ainsi de "partager » la contribution en plusieurs lieux simultanément.

Ainsi, nous définirions les "pratiques transplateformes » comme un usage d'Internet où les contributions se déclinent sur diverses plateformes Web, où le contributeur partage un contenu dans un site, puis dans un ou plusieurs autres, par des manipulations logicielles automatisées ou non, qui sont souvent intégrées dans l'interface même des sites (notamment Facebook et Twitter). Il s'agit par exemple d'un usager qui crée un podcast, le met en ligne sur son blog, le publicise sur Twitter et sur Facebook, y joint des images qui se retrouvent sur Flickr, etc. Conséquemment, certains commentateurs de ce podcast s'exprimeront sur le blog du podcaster, d'autres le feront directement sur Twitter ou sur Facebook. Les usagers que nous avons observés ont développé des stratégies pour étendre leur auditoire grâce à ces pratiques transplateformes. Ainsi, le « fil » d'une communication sera réparti sur diverses plateformes. Si par ces pratiques les podcasters rejoignent ainsi un plus grand nombre de gens, cela ne va pas sans avoir certaines conséquences.

\section{Suivre les traces et l'enchevêtrement des usages : le cas des podcasters Lada et Georges}

Les podcasts recèlent de marqueurs identitaires forts et la signature stylistique d'un podcaster est reconnaissable : la voix, le ton, le style d'animation, la musique et les sujets abordés sont autant de marqueurs qui permettent au podcaster de se distinguer. Cette facette sonore est presque toujours articulée à des textes et à des photographies qui contribuent à compléter le podcast. Dans le podcasting indépendant, la communication avec l'auditeur est profondément marquée par les pratiques transplateformes. Il émerge de nos observations préliminaires que deux grandes logiques de pratiques transplateformes se déploient dans le podcasting. Cependant, elles ne sont pas exclusives et des tendances médianes sont aussi observables:

\section{Le relayage}

Il s'agit ici de rediffuser un contenu sur plusieurs plateformes, par exemple l'annonce d'un nouvel épisode du podcast. Cette pratique très répandue, que 
l'on pourrait associer aux stratégies cross-médias mentionnées plus haut, semble viser à accroître la visibilité et la notoriété du podcast afin d'en étendre l'auditoire.

\section{La déclinaison}

Ce type de pratiques consiste à diffuser une série de contenus apparentés, mais distincts et complémentaires, sur diverses plateformes. Un cas typique sera de lancer un nouvel épisode du podcast, de l'accompagner d'un billet de blog, de photos dans Flick ou Instagram ${ }^{7}$ et d'un statut sur Facebook où on inscrira le lien vers le podcast ainsi qu'un commentaire personnel appréciatif, par exemple « J'aime particulièrement la chanson qui ouvre le podcast de cette semaine ". Il en résulte un enchevêtrement complexe, où pour suivre le fil d'une communication il faut savoir lire les traces laissées par les pratiques transplateformes. D'un point de vue méthodologique, mais aussi au niveau de la compréhension de ces traces, il devient périlleux d'aligner ces fragments, comme nous allons en faire la démonstration.

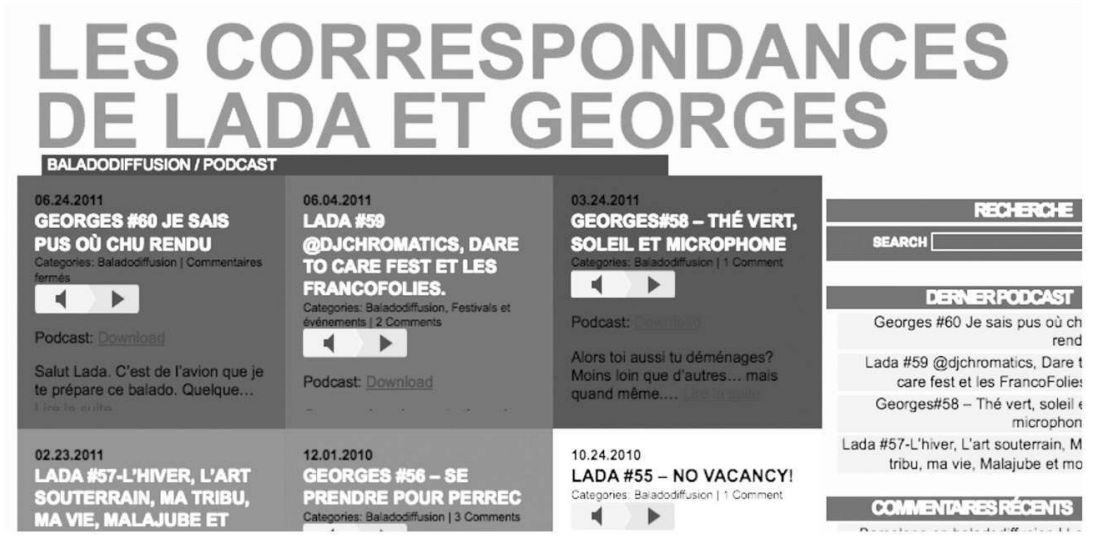

Figure 1 : Page d'accueil du podcast Lada et Georges.

Source : http://www.ladaetmoi.com/

La Figure 1 montre la page d'accueil du blog du podcast indépendant Lada et Georges, une «balado-correspondance » où deux amis de longue date se dédient publiquement un podcast, à tour de rôle, sous les pseudonymes de Lada et de Georges. Nous allons nous attarder au cas de Georges, en remontant le fil des pratiques transplateformes liées au podcast \#60, identifié par la flèche (fig. 1). En cliquant sur le carré pointé par la flèche, on arrive au billet qui présente cet épisode du podcast (fig. 2). Comme on le voit

7 Réseau socionumérique qui fonctionne principalement à partir d'une application mobile pour téléphone intelligent et qui permet le partage de photographies, jumelées à un système de géo-localisation et à un traitement de l'image de style vintage. Notez que depuis la rédaction de cet article, Instagram a été acheté par Facebook. 


\section{C\&O n ${ }^{\circ} 43$}

dans la capture d'écran (fig. 2), le bouton « Like » de Facebook et l'option de commenter le billet de blog sur Facebook sont offerts à l'usager en bas du texte. Lintégration de ces boutons à même le blog invite les auditeurs à publiciser le podcast sur Facebook en y ajoutant un marqueur symbolique de leur appréciation, soit le «Like » du site de réseautage, soit un commentaire personnel qui sera simultanément diffusé à tous leurs " amis " Facebook. Notez que ces possibilités sont offertes sur le blog des podcasters, qui est ici opéré à partir de la plateforme de logiciel libre Wordpress.

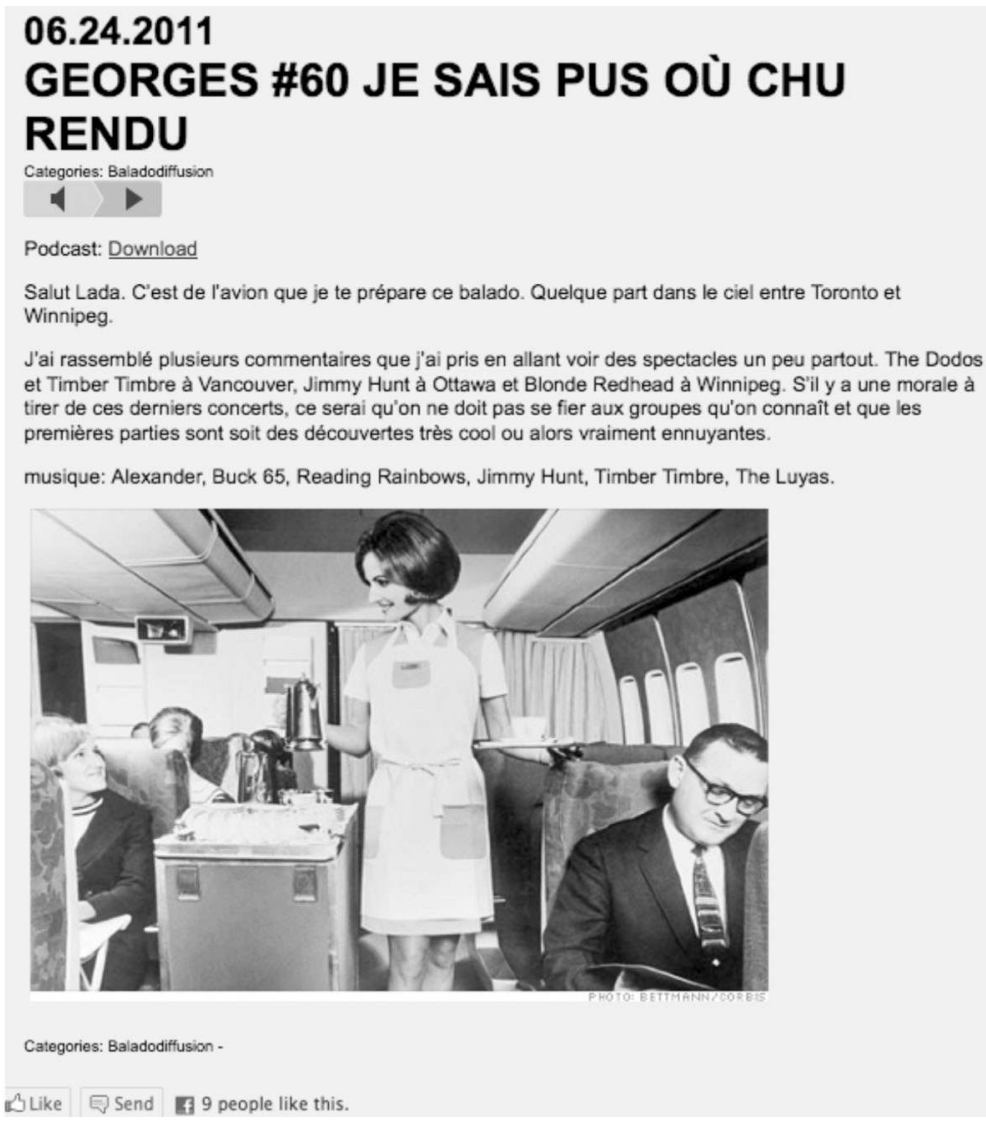

Figure 2 : Billet de blog présentant le podcasł\#60 de Lada et Georges. Source : http://www.ladaetmoi.com/

En plus de leur blog, Lada et Georges possèdent une page Facebook publique dédiée à leur podcast ${ }^{8}$. Ils y ont publié le titre et le lien du podcast \#60 afin de le diffuser aux usagers qui ont adhéré à cette page. Mais mis à 
part des usagers qui ont « aimé » cette nouvelle, il n'y a aucun commentaire sous cette publication dans leur page Facebook. Cela ne signifie pas que la conversation se soit tarie pour autant.

C'est plutôt sur les profils personnels des deux podcasters que la communication s'est déplacée, changeant par le fait même de registre et passant du public (l'usage de pseudonymes, la page officielle du podcast sur Facebook) à l'intime (les véritables noms des podcasters, leurs comptes personnels sur Facebook). Suivons la conversation sur le profil personnel de George.

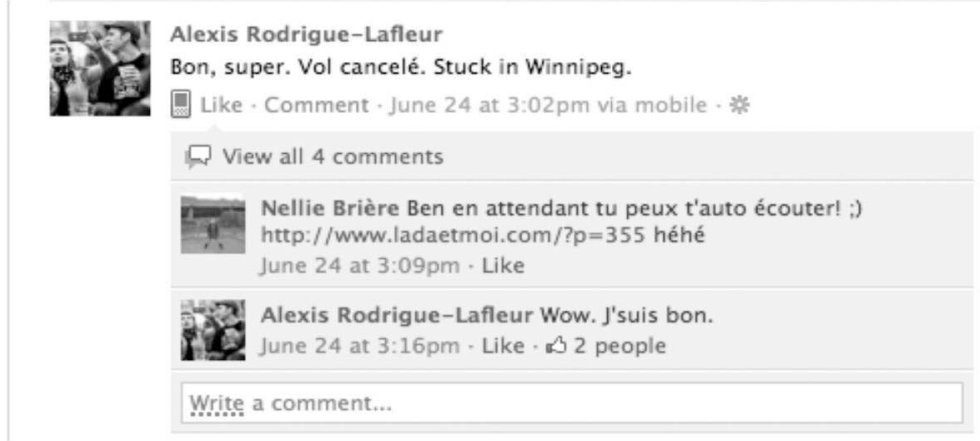

Figure 3 : Mention du podcast \#60 de Lada et Georges dans les commentaires liés à un statut Facebook de l'un des podcasters. Source : facebook. com/[nom de l'usager]

L'une des ramifications entourant la diffusion du podcast \#60 surgit après quatre commentaires personnels liés au statut de Georges, qui a des problèmes de transport aérien et qui est en attente dans un aéroport canadien (fig. 3) ${ }^{9}$. Suite à ces commentaires, l'usagère derrière le pseudo "Lada ", dont le commentaire est identifié par la flèche, propose à son acolyte d'écouter son propre podcast en attendant son vol et lui partage le lien pour l'écouter sur leur blog. Ce à quoi Georges répond ironiquement "Wow. J'suis bon »- commentaire que deux autres usagers « aiment ». En passant la souris sur cette mention, une boîte apparaît et donne les noms des personnes qui aiment le commentaire en question, dans ce cas-ci Lada et un autre usager reconnu comme étant un auditeur du podcast.

Le fragment partagé ici permet de dessiner les contours d'un Georges qui est en train d'effectuer un voyage au Canada, plus précisément à Winnipeg, et au cours duquel il a saisi l'occasion de voir des spectacles de musique émergente. Une investigation plus approfondie montre que les spectacles vus lors de ce voyage forment la trame musicale du podcast \#60. Les interactions

9 Nous avons anonymisé les noms des usagers par souci de confidentialité. 
avec Lada sur la page Facebook personnelle de Georges laissent entrevoir un réseau de médiations de soi entourant Georges et où les traces vont du podcast, au blog puis au site Facebook et même entre les profils personnels et publics des podcasters. Notons que des liens vers d'autres plateformes restent à creuser dans ce cas-ci, notamment vers iTunes où le podcast est disponible, et les profils Blip. fm et Instagram de Georges, pour ne nommer que ceux-là.

\section{Traces et convergence}

Le cas de la diffusion du podcast \#60 de Lada et George montre bien l'enchevêtrement des traces médiatiques issues des pratiques transplateformes. Internet permet de détacher le fond et la forme des informations pour les réassembler de multiples manières. Désarticulées, ces données sont ainsi agencées au fil des usages et des demandes, facilement transférables d'un blog à Facebook, à Twitter et ainsi de suite, laissant des traces au fur et à mesure qu'elles sont sollicitées.

Importé de la sémiotique et de l'anthropologie, le concept de trace médiatique prend une dimension particulière avec le contexte des réseaux socionumériques actuels et des pratiques transplateformes que nous décrivons. «Dans la culture numérique, le signe, le message et le document sont appelés à être subsumés dans la catégorie des traces. Celle-ci ne désigne pas un nouveau type d'objet, mais un mode inédit de présence et d'efficacité, lié aux caractéristiques techniques et sociales des réseaux. " (Merzeau 2009). Dans l'épaisseur des pratiques numériques, le concept de trace prend donc deux dimensions distinctes. D'abord celle, classique, d'un signe témoignant d'un passage, telle une marque de pas dans la neige, et chargé de significations modulées selon l'encyclopédie personnelle du lecteur de ce " texte » (Eco 1979). Ensuite, celle d'un fil d'Ariane extensible à l'infini, pour toute donnée qui circule en ligne, dont la racine est nouée aux logiques de traçabilité des systèmes informatiques, dont l'adresse IP des usagers (Perriault2009).

L'intégration de modalités de partage transplateforme aux médias sociaux vient supporter ces pratiques en les facilitant. Dans la figure 4 ci-après, on reconnaît un statut Facebook tiré du profil du podcaster Georges. Ce statut, où Georges partage une image d'un spectacle du groupe de musique Pâte filo, a été initialement diffusé sur la plateforme Instagram. Pour connaître la provenance de la contribution, il faut savoir interpréter la trace de la pratique transplateforme : ici, la mention « via Instagram » qui apparaît en bas de la photo après la date. L'architecture des plateformes et le design des interfaces ne sont pas neutres et contribuent donc à la manière dont s'opèrent les pratiques transplateformes, notamment en intégrant des boutons de partage directement dans les interfaces, mais aussi par la façon dont elles sont décodés et reçues. 


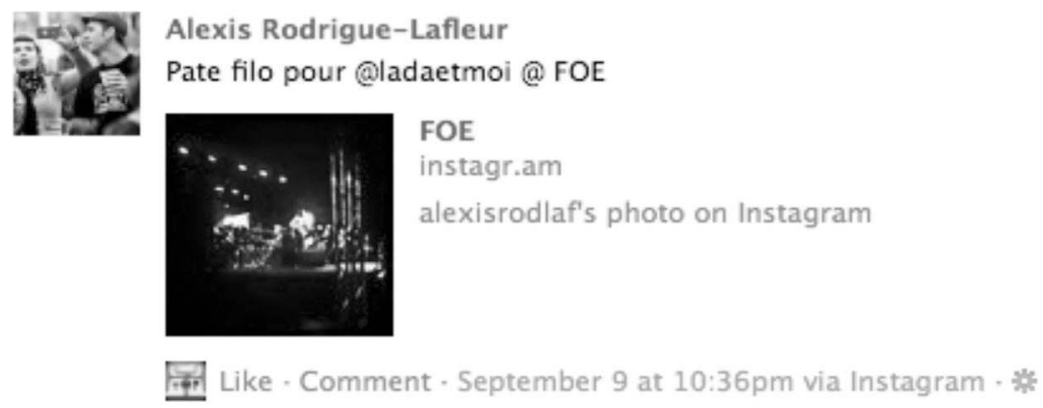

Figure 4 : Pratiques transplateformes d'Instagram à Facebook. Source : facebook. com/[nom de l'usager]

Cet exemple illustre une tendance que nous observons dans nos recherches quant à une convergence vers Facebook des contenus publiés par les podcasters dans les médias sociaux. Or, notre expérience d'usagère de plusieurs plateformes nous permet de penser que cette tendance gagne en popularité. Facebook « colonise " graduellement les plateformes du Web social, où il est désormais commun de retrouver le bouton bleu marqué d'un « F » témoignant de la tendance impérialiste de ce géant américain. La plupart des grandes plateformes de blogging (dont Blogger et Wordpress), de même que Twitter, Blip. fm, Instagram, Flickr et la plateforme vidéo Vimeo ont désormais un bouton de rediffusion vers Facebook. Même YouTube, pourtant l'une des possessions phares de Google, champion de l'impérialisme numérique (Proulx et Millette 2010) et principal concurrent de Facebook, a intégré le bouton bleu à sa plateforme.

\section{Gestion des pratiques transplateformes et dissonance}

Cette convergence des contenus Facebook a plusieurs implications. Par exemple au niveau des publics auxquels on s'adresse. L'usage de Facebook, en tant que tel, implique une décontextualisation et un aplanissement des divers publics et rôles sociaux d'une personne et ce, malgré les tentatives renouvelées du site pour permettre aux usagers de gérer la visibilité de leurs échanges (boyd 2007 ; 2010). Si l'on juxtapose cela aux pratiques transplateformes qui tendent à relayer sur Facebook des contenus auparavant créés ailleurs, on ajoute à la confusion entre les divers publics auxquels on s'adresse.

Il devient difficile de cerner les implications de ces pratiques en termes de visibilité et de diffusion même si, comme c'est le cas chez les podcasters, les usagers orchestrent leurs communications finement. L'un des acteurs interrogés récemment, qui figure comme le plus actif au niveau des pratiques transplateformes parmi nos informateurs, affirmait d'ailleurs se préoccuper d'éviter de créer de la « dissonance » par rapport à son image dans les médias sociaux, tout en étant très appliqué à ne pas trop « se répéter » d'une plateforme 
à l'autre. On peut aisément imaginer la gestion minutieuse nécessaire à la création et la diffusion de contenus de cet usager soucieux d'offrir une image cohérente à ses publics, sans les saturer de redites, mais tout en relayant quasi systématiquement vers Facebook ses publications depuis Twitter, Blip et Instagram.

Cependant, les podcasters ne représentent qu'une frange des internautes, probablement parmi les plus compétents en termes techniques et communicationnels par rapport aux usagers des médias sociaux en général. Nous avons déjà montré ailleurs (Millette) que les podcasters sont en effet très souvent des usagers « experts » qui, sans être des professionnels des médias ou de la diffusion sonore en ligne, ont des compétences pointues dans un ou plusieurs des domaines de l'informatique, des communications et des médias. Munis de telles compétences, les podcasters sont probablement mieux outillés décoder les contenus issus pratiques transplateformes. Pour l'usager lambda, ces pratiques pourraient cependant s'accompagner de dissonance, ne seraitce que par rapport aux écarts de registres communicationnels et normatifs qui se produisent lorsque l'on transpose un message d'une plateforme à une autre. Par exemple, un message d'abord diffusé sur Twitter est souvent court et suit une logique narrative axée sur le faire à cause de la contrainte de 140 caractères inhérente à la plateforme (Cardon 2008 : 119). Un tel message, une fois rediffusé sur Facebook, pourrait paraître abrupt pour un public qui ne saurait décoder les traces indiquant qu'il s'agit du relayage d'un tweet.

\section{Conclusion}

Nous avons ici formulé une première tentative pour combler un point aveugle dans les travaux sur les médias sociaux et les usages d'Internet, soit la présence de plus en plus marquée de pratiques transplateformes. Pour mener notre réflexion, nous avons théorisé, explicité et analysé ces pratiques en mobilisant des données tirées d'une étude de cas sur le podcasting indépendant. Comme tendent à le montrer nos observations actuelles, les pratiques transplateformes semblent faire converger les contenus vers le réseau socionumérique Facebook.

$\mathrm{Du}$ point de vue de la méthodologie, ces pratiques transplateformes appellent le chercheur à faire preuve d'une grande créativité dans ses techniques de collecte. Suivre le fil des communications relève presque de la traque. Éthiquement parlant, cela soulève aussi plusieurs questions, puisque le fil d'une communication peut facilement glisser d'une plateforme publique vers une plateforme où l'internaute étudié a développé un usage plus personnel et intime. Il nous apparaît ainsi qu'au-delà des méthodes, les pratiques transplateformes demandent au chercheur de questionner sa transparence envers les sujets de son enquête. De plus, les pratiques transplateformes et de la convergence qui en découlent ont des implications en termes de vie privée. Quel statut revêt une donnée partagée de manière limitée sur une 
plateforme À lorsqu'on la relaie sur une plateforme B dont les paramètres de sécurité sont plus lestes ? Et qu'en est-il de ces traces qui contribuent à enrichir les bases de données de toutes ces tierces applications au fil de nos pratiques transplateformes ${ }^{10}$ ? Ces questions témoignent de la complexité que représentent les usages des médias sociaux pour la recherche en sciences sociales. Nous comptons poursuivre nos activités afin de contribuer à éclaircir quelques-uns de ces questionnements.

\section{BIBLIOGRAPHIE}

BOUQUILLION P., JACOB T. M., Le Web collaboratif-Mutation des industries de la culture et de la communication, Grenoble, PUG, 2010.

BOYD D., «Facebook and 'radical transparency' (a rant) », Billet de blog sur Apophenia, 2010, http://www.zephoria.org/thoughts/archives/2010/05/14/facebook-and-radicaltransparency-a-rant.html [Consulté septembre 28, 2011].

BOYD D., "Social Network Sites : Public, Private, or What ? ", The Knowledge Tree, 2007, Disponible en ligne : http://kt.flexiblelearning.net.au/tkt2007/?page_id=28

BROOKER W., "Living on Dawson's Creek ", International Journal of Cultural Studies, Vol. 4, No4, 2001, p. 456 -472.

CARDON D., "Le design de la visibilité. Un essai de cartographie du web 2.0 ", Réseaux, 269 (152), 2008, p. 93-137.

ECO U., Lector in fabula - Le rôle du lecteur ou la coopération interprétative dans les textes narratifs, Paris, Grasset, 1979.

EVANS E. J., "Character, audience agency and transmedia drama », Media, Culture E Society, Vol. 30 №2, 2008, p. 197 -213.

JENKINS H., Convergence Culture - Where Old and New Media Collide, NYU Press, New York-Londres, 2006a.

JENKINS H., Fans, Bloggers and Gamers - Exploring Participatory Culture, NYU Press, New York-Londres, 2006b.

MERZEAU L., " Du signe à la trace : l'infomation sur mesure ", Hermès, №53, 2009, p. 23-29, Disponible en ligne : http://halshs.archives-ouvertes.fr/view_by_ stamp. php ?\&halsid=40tc98913kle31pf88se2796a1\&label=SHS\&langue=fr\&action todo=view\&id=halshs-00483292\&version $=1$

MILLETTE M., Usages contributifs sur Internet : le podcasting indépendant et le sens de son style, Mémoire de maîtrise, (dir.) Serge PROULX, Université du Québec à Montréal, 2009, Disponible en ligne : http://uqam.academia.edu/M \%C3 \%A9lanieMillette/ Papers/642928/Usages_contributifs_sur_Internet_le_podcasting_independant_et_le_ sens_de_son_style

PERRIAULT J., "Traces (numériques) personnelles, incertitude et lien social », Hermès, №53, 2009, p. 13-20.

10 En effet, pour qu'un relayage d'une plateforme $\mathrm{A}$ à $\mathrm{B}$ ait lieu, l'usager doit accepter de partager les données de son compte $\mathrm{B}$ avec la plateforme $\mathrm{A}$ à partir de laquelle le message sera rediffusé. 
PERRYMAN N., "Doctor Who and the Convergence of Media », Convergence: The International Journal of Research into Nerw Media Technologies, Vol. 14 №1, 2008, p. 21-39.

PROULX S., MILLETTE M. et LORNA H., Médias sociaux : Enjeux pour la communication, PUQ, Québec, 2012.

PROULX S., MILLETTE M., " Limpérialisme numérique américain ", in BADIE B., VIDAL D., La fin du monde unique 50 idées-forces pour comprendre l'état du monde (édition 2011), Paris, La Découverte, 2010.

Résumé : Cet article est une première réflexion qui vise à combler un point aveugle dans les travaux sur les usages d'Internet, soit la présence de plus en plus marquée de pratiques transplateformes dans les médias sociaux. Pour se faire, nous mobilisons notamment des ancrages théoriques en sociologie des usages et en Media Studies afin de mener une analyse de ces pratiques transplateformes dans les médias sociaux. Ancré dans des données issues d'une étude de cas sur le podcasting, cet article expose une tendance quant à la convergence des contenus publiés dans les médias sociaux vers le site de réseautage social Facebook.

Mots-clés : Facebook, Médias sociaux, Podcasting, Pratiques transplateformes, Présentation de soi.

Abstract : This paper reports some preliminary work that seeks to fill a blind spot in the literature of Internet uses, namely the multiplication of transplatform practices in social media. I mobilize a conceptual frame in usages sociology and Media Studies to understand those practices through the lens of self-presentation in social media. Drawing from data from a case study among independent podcasters, I find a trend in social media, where content converge towards the social network platform Facebook.

Keywords : Facebook, Podcasting, Self Presentation, Social Media, Transplatform Practices. 